\title{
Relation of transition region blinkers to the low chromosphere
}

\author{
A. Brković and H. Peter \\ Kiepenheuer-Institut für Sonnenphysik, Schöneckstr. 6, 79104 Freiburg, Germany \\ Received 12 March 2003 / Accepted 12 May 2003

\begin{abstract}
We investigated the coupling between the low chromosphere, lower and upper transition region through the study of blinkers. Blinkers are extreme ultraviolet (EUV) small-scale transient brightenings best detected in transition region lines showing an intensity enhancement on the 60-100\% level on average. We analysed time-series spectra of the $\mathrm{C}_{\text {II }} 1037 \AA(2-4 \times$ $10^{4} \mathrm{~K}$, lower transition region) and $\mathrm{O}$ VI $1038 \AA\left(3 \times 10^{5} \mathrm{~K}\right.$, upper transition region) lines, as well as the $\mathrm{C}_{\mathrm{I}}$ continuum at $1042 \AA$ formed about $1 \mathrm{Mm}$ above the solar surface, obtained with the Solar Ultraviolet Measurement of Emitted Radiation (SUMER) spectrometer on SOHO. Blinkers detected in $\mathrm{C}_{\text {II }}$ and $\mathrm{O}_{\text {VI }}$ have similar enhancement levels. We found a modest space-time overlap between blinkers in the two lines and a small correlation between $\mathrm{C}_{\text {II }}$ or $\mathrm{O}_{\mathrm{VI}}$ blinkers and the corresponding light curve in the chromospheric continuum. This suggests a relation of EUV blinkers to the chromosphere, which could support the idea that blinkers are the consequence of granular collisions. The average duration of $\mathrm{O}$ vi blinkers amounts to 10.0 min and of $\mathrm{C}_{\text {II }}$ blinkers to $6.7 \mathrm{~min}$.
\end{abstract}

Key words. Sun: chromosphere - Sun: corona - Sun: transition region - Sun: UV radiation

\section{Introduction}

Since the first reports on blinkers by Harrison (1997) and Harrison et al. (1999) studies of the solar transition region were very often coupled with analyses of these brightening events. Harrison introduced the term "blinker" to describe EUV brightenings observed in the quiet Sun lasting on average about $1000 \mathrm{~s}$. He reported signficant brightness enhancements in the transition region line of O IV $554 \AA\left(1.6 \times 10^{5} \mathrm{~K}\right)$. Harrison et al. analysed simultaneous observations of the chromospheric He I $584 \AA$ A line $\left(2 \times 10^{4} \mathrm{~K}\right)$, the transition region lines O III $599 \AA\left(10^{5} \mathrm{~K}\right), \mathrm{O}_{\text {IV }} 554 \AA$ and O v $629 \AA\left(2.5 \times 10^{5} \mathrm{~K}\right)$ and the coronal $\mathrm{Mg}_{\mathrm{IX}} 368 \AA$ and $\mathrm{Mg} \times 624 \AA$ lines $\left(10^{6} \mathrm{~K}\right)$. That study showed that blinkers are mainly located in the network, and are not due to a temperature increase of the plasma, but are probably caused by density enhancements or changes in brightness filling factor. Significant increases in brightness in chromospheric and coronal lines have not been reported in the study. Based on this result Harrison et al. concluded that blinker activity is specific only for transition region temperatures. Brković et al. (2001) analysed simultaneous quiet-Sun observations of $\mathrm{He}, \mathrm{OV}$ and $\mathrm{Mg} \mathrm{IX}$ lines and found significant flux enhancements in the He I line as well. Brković et al. detected blinkers both in the network and the inter-network. Bewsher et al. (2002) also analysed quiet-Sun observations and reported that blinkers are approximately located above regions

Send offprint requests to: A. Brković, e-mail: alen@kis . uni-freiburg.de of strong unipolar field. Parnell et al. (2002) studied blinkers in active regions and found very similar results to the quietSun blinker studies. The difference is reflected through somewhat larger enhancements and frequency of occurrence in active regions.

Blinkers are just one of several phenomena characterising the solar transition region. Another type of transient events observed in EUV transition region lines are explosive events (e.g., Brueckner \& Bartoe 1983; Dere et al. 1984; Innes et al. 1997). Their main characteristics are spatial sizes of several arcsecs and highly broadened line profiles with line shifts of order $100 \mathrm{~km} \mathrm{~s}^{-1}$. Explosive events are sometimes, but not necessarily, connected to a strong brightening and were detected in the network and the inter-network. Their lifetime is up to $100-150 \mathrm{~s}$. The coronal and chromospheric signatures of explosive events have recently been discussed, as follows: analysing the $\mathrm{Fe} \mathrm{Ix} / \mathrm{Fe} \times 171 \AA\left(10^{6} \mathrm{~K}\right)$ TRACE (Transition Region and Coronal Explorer; Handy et al. 1999) images of an active region together with SUMER C Iv $1548 \AA\left(10^{5} \mathrm{~K}\right)$ rasters, Winebarger et al. (2002) found a modest signature of explosive events in the corona. They argued that this result could also be due to the transition region O vi $172 \AA$ $\left(3 \times 10^{5} \mathrm{~K}\right)$ line which is relatively weak when compared with the Fe Ix/Fe x 171 A lines. Teriaca et al. (2002) applied SUMER to search for the coronal signature in the $\mathrm{Mg} \times 624 \AA$ line during explosive events observed in the $\mathrm{N} v 1238 \AA\left(1.8 \times 10^{5} \mathrm{~K}\right)$ line and did not find any signs of these, neither in the line profile nor in its integrated intensity. On the other hand, they found the 
response of the upper chromosphere observed in the $\mathrm{S}_{\text {II }} 1253 \AA$ $\left(3.5 \times 10^{4} \mathrm{~K}\right)$ line to explosive events in Nv. Madjarska \& Doyle (2002) also applied SUMER and found that the emission in $\mathrm{H}_{\text {I }}$ Ly $6^{1} 930 \AA$ is correlated to explosive events detected in $\mathrm{S}$ VI $933 \AA\left(1.9 \times 10^{5} \mathrm{~K}\right)$.

The relationship between the blinkers and explosive events is not yet clear. Since these events were detected with different instruments and their observational characteristics are different it is not easy to give a final answer on this question. Some explosive events show increase in brightness on the order of a factor three and more and would be detected as blinkers as well. In principle, it is possible that some, specially shorter living, blinkers are explosive events. Chae et al. (2000) compared simultaneous observations of explosive events obtained with SUMER (Wilhelm et al. 1995) and of blinkers detected using the Coronal Diagnostic Spectrometer (CDS; Harrison et al. 1995). They showed that explosive events tend to occur on the edges of, and simultaneous with, blinkers. Another similar study is the one of Innes (2001) who compared SUMER observations to EUV images from TRACE. Her results confirmed that the $\mathrm{C}$ IV transition region line from TRACE exhibits brightenings in association with velocity events. These results of Chae et al. (2000) and Innes (2001) suggest a close relationship between the blinkers and explosive events, but this statement is far from being proven and needs further investigation.

It is very important to understand the connection between the underlying photospheric magnetic field and transients such as blinkers and explosive events. Having understood this connection one could eventually properly place the role and importance of different mechanisms (e.g., reconnection, flow through the loop driven by asymmetric heating) for these transients. Harrison et al. (1999) proposed that a blinker is the result of a reconnection between newly emerging flux and the network. The result of Bewsher et al. that blinkers are approximately located above regions of strong unipolar field, rejects magnetic reconnection as the process causing blinkers unless unresolved opposite polarity flux is present. Bewsher et al. used the Michelson Doppler Imager (MDI; Scherrer et al. 1995) onboard SOHO for obtaining this result. Since the pixel size of MDI is $2^{\prime \prime}$ it can easily be the case that some, if not a lot, of bipolar magnetic flux exists in the MDI resolution element which remained unresolved. Tarbell et al. (2000) analysed simultaneous observations obtained with TRACE, MDI and SUMER and found that the enhanced emission in the transition region $\mathrm{C}$ Iv line is generally co-spatial with the magnetic pattern in the photosphere. They determined a good correlation of the appearance of bright transients and cancelling mixed polarity magnetic elements in the photosphere, but they also detected transients in regions where no fields were detected with the MDI sensitivity. Tarbell et al. reported that blinkers and other transient brightenings were often accompanied with bi-directional jets. Based on these results they proposed that a basic process causing energetic events, such as blinkers or explosive events, is the cascade of shock waves produced by colliding and reconnecting flux tubes. They suggested that the

\footnotetext{
${ }^{1}$ According to Fontenla et al. (1993) H HLy 6 is formed in the uppermost part of the chromosphere above $9000 \mathrm{~K}$.
}

Table 1. Observational details.

\begin{tabular}{ccccc}
\hline \hline $\begin{array}{c}\text { Date } \\
(\mathrm{UT})\end{array}$ & $\begin{array}{c}\text { Start } \\
\text { time }\end{array}$ & $\begin{array}{c}\text { End } \\
\text { time }\end{array}$ & $\begin{array}{c}\text { Cadence } \\
(\mathrm{s})\end{array}$ & $\begin{array}{c}\text { Slit No. } \\
\left(\operatorname{arcsec}^{2}\right)\end{array}$ \\
\hline $97-04-25$ & $02: 05$ & $05: 10$ & 16 & $4(1.0 \times 120)$ \\
$98-05-12$ & $14: 36$ & $16: 02$ & 12 & $8(0.3 \times 120)$ \\
$98-05-16$ & $14: 30$ & $16: 40$ & 12 & $2(1.0 \times 300)$ \\
\hline
\end{tabular}

converging supergranular flows could play very important roles in the coupling between the photosphere and chromosphere and transition region.

Finally, Priest et al. (2002) proposed models for the origin of blinkers. They suggested five mechanisms which could eventually produce blinkers. The mechanisms include: the heating of cool spicular material; the containment of plasma in low-lying loops in the network; the thermal linking of cool and hot plasma at the feet of coronal loops; the heating and evaporating of chromospheric plasma in response to a coronal heating event; and the cooling and draining of hot coronal plasma when coronal heating is switched off. In each case a blinker could be produced when a network junction is being compressed more than normal by granules. Their scenarios still need to be tested observationally and this paper is one attempt in this direction.

Our goal is to investigate the connection of transition region blinkers to the lowest layer of the chromosphere in the EUV. To establish such a connection one should not only use EUV emission lines, as they are formed in the upper chromosphere and above, but one has to rely on the continua formed just at and above the temperature minimum (Vernazza et al. 1981). To perform this study we will use the SUMER spectrograph on SOHO, because this allows us to study not only lines formed at transition region temperatures, but also continua from the chromosphere. Here we will concentrate on a spectral window including $\mathrm{O}$ VI 1038 from the upper transition region $\left(3 \times 10^{5} \mathrm{~K}\right)$, $\mathrm{C}_{\text {II }} 1037$ from the lower transition region $\left(2-4 \times 10^{4} \mathrm{~K}\right)$ and the $\mathrm{C}_{\text {I continuum near }} 1042$ formed in the lowest layer of the chromosphere about $1 \mathrm{Mm}$ above the solar surface $\left(\tau_{5000}=1\right)$.

\section{Observations}

We analysed SUMER data of SOHO Joint Observing Program (JOP) 20 and JOP 72. Dates of observations, exposure times and slits used are given in Table 1. Quiet-Sun regions near the disc centre were observed with SUMER's detector B and the slit was kept at a fixed location on the solar surface by compensating for solar rotation. The widths of spectral windows were about $2.2 \AA$.

Prior to the analysis several instrumental corrections have been applied. For the flat-field correction we used the flat-field images taken on 24 April 1997 and 19 February 1998. The pincushion distortion of the image and the inclination of the spectral lines with respect to the detector columns were removed. The effects of the dead time and gain depression of the detector were almost negligible, but the corrections have been applied anyway. After applying these corrections we applied double Gaussian fits to the spectral window containing $\mathrm{C}_{\text {II }}$ and $\mathrm{O}$ VI, 

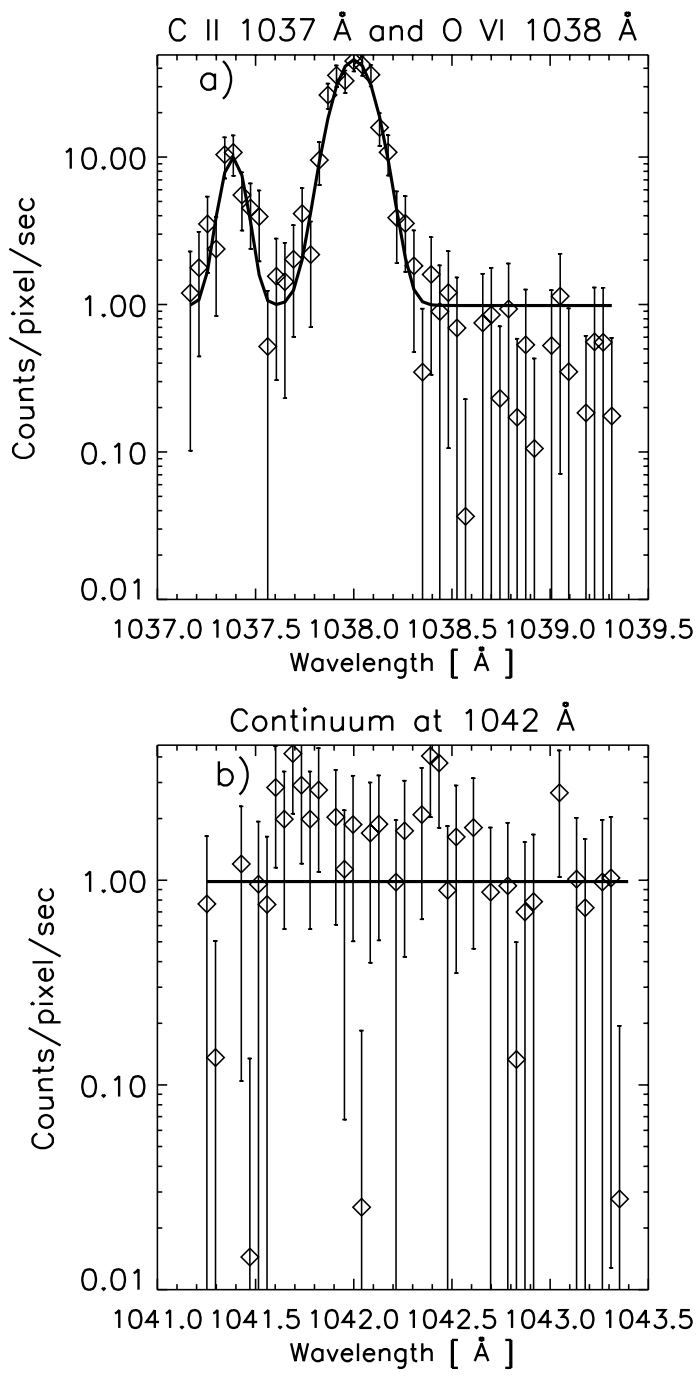

Fig. 1. Sample spectra of the $\mathrm{C}_{\text {II }}$ and $\mathrm{O}_{\mathrm{VI}}$ lines a), as well as the neighbouring $\mathrm{C}_{\mathrm{I}}$ continuum near $1042 \AA$ b). The observations are shown as bars that represent the (Poisson) errors of the count rate. The double Gaussian fit to the two lines (panel a) and the continuum calculated from the continuum window (panel b) are shown as thick solid lines.

thus separating the two lines. Least-squares fits were applied and a linear background was subtracted in the fits. The fitting procedure used a Genetic Algorithm based optimisation method of Charbonneau (1995) because it provides us with a reliable tool for spectral fitting (McIntosh et al. 1998). For the evaluation of the continuum we used a separate spectral window simultaneously observed with $\mathrm{C}_{\text {II }}$ and $\mathrm{O}$ vi and summed over spectral pixels. Figure 1 shows an example of the fitting procedure. Since the absolute calibration of wavelengths was not necessary for this study we did not perform it. Figure 2 shows space-time diagrams of the continuum near $1042 \AA$, $\mathrm{C}_{\text {II }}$ and O VI obtained on 25 April 1997. The $\mathrm{C}_{\text {I }}$ continuum is formed in the lowest chromosphere at $\approx 4500 \mathrm{~K}$, i.e., just above the temperature minimum according to Vernazza et al. (1981), $\mathrm{C}_{\mathrm{II}}$ is formed over a broad range of temperatures $2-4 \times 10^{4} \mathrm{~K}$, $\mathrm{O}$ VI at $3 \times 10^{5} \mathrm{~K}$ (both temperatures represent a peak of the

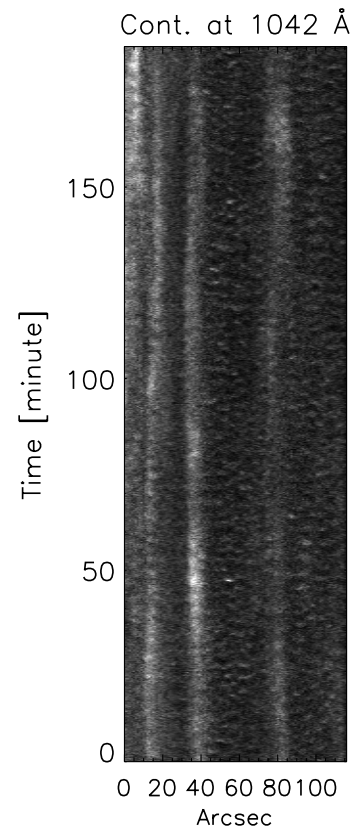

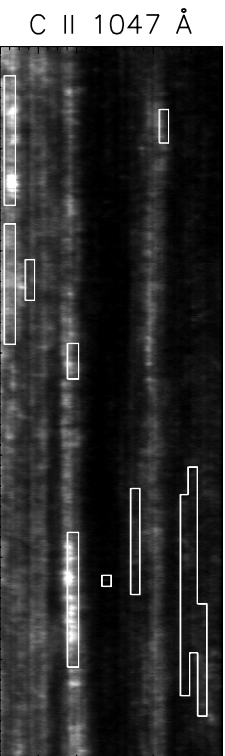

020406080100 Arcsec
O VI $1038 \AA$

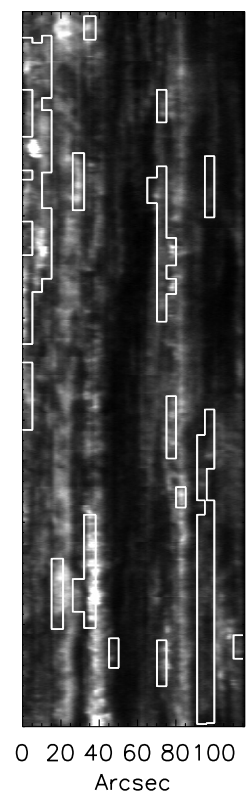

Fig. 2. Space-time diagrams of the continuum, $\mathrm{C}_{\text {II }}$ and $\mathrm{O}$ vi recorded on 25 April 1997 with SUMER. The intensities are plotted on a logarithmic scale. Regions of bright network can be seen near 20,40 and 80 arcsec. The contours on the middle and the right frame depict all the blinkers present at some time during the three hours of observations.

ion's relative abundance according to Arnaud \& Rothenflug 1985). Therefore, this data set samples the solar atmosphere from the low chromosphere to the upper transition region. In this paper we show the analysis of intensities, while $\mathrm{C}_{\text {II }}$ and O vi line widths and line shifts together with the corresponding intensities are part of another study.

\section{Blinker identification}

The first step was to identify the blinkers in $\mathrm{O}$ VI and $\mathrm{C}$ II. For this purpose we used the same automated routine as applied by Brković et al. (2001). The routine was originally developed to detect transition region brightenings classified in CDS-studies as blinkers. To investigate relation of blinkers to the chromosphere we wanted to use similar thresholds as in the CDS case because blinkers have only been investigated with CDS, so far. Since SUMER reveals higher levels of variability (Brković et al. 2002) we averaged data over five to ten spatial pixels and thus reduced SUMER spatial resolution in order to match the one of CDS. The reduction in the spatial resolution does not affect the final general results, and at the same time results, e.g. spatial locations and durations, can be compared to the results obtained with CDS. The difference in the number of pixels over which the averaging was performed was due to different brightening levels in analysed data sets. We also took care to average over regions of similar brightness, i.e., not artificially mixing bright and dark regions. Now, we proceed with the description of the blinker identification method keeping in mind that a pixel is actually a group of spatially averaged pixels of the SUMER detector. 
O VI $1038 \AA$

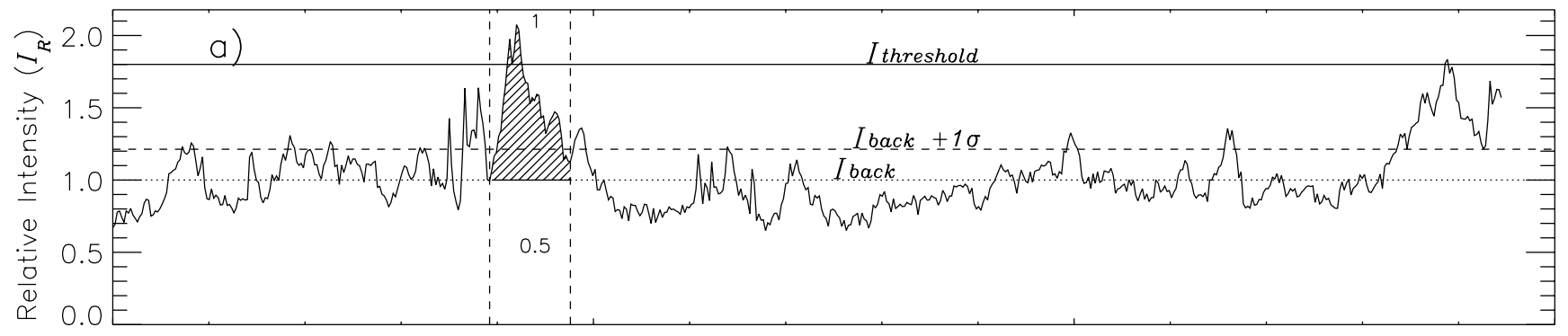

C $111037 \AA$

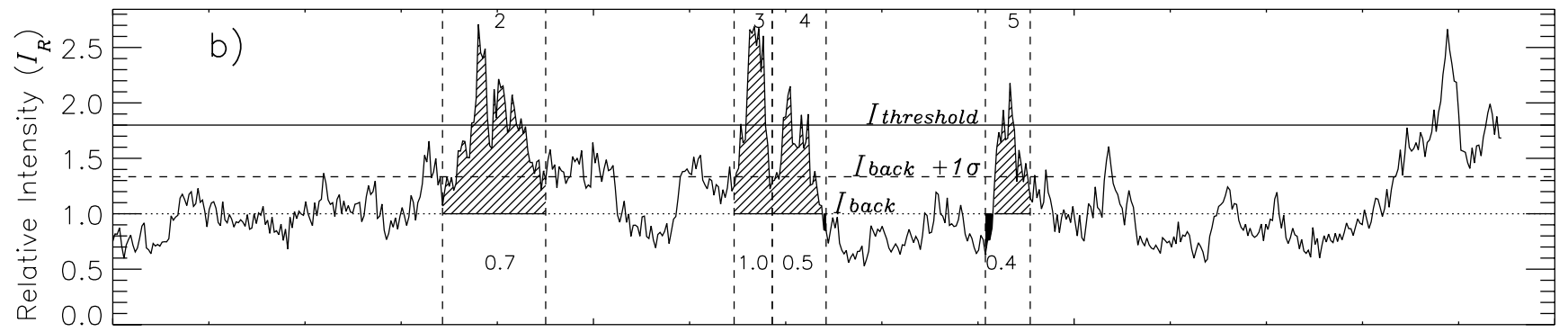

Continuum at $1042 \AA$

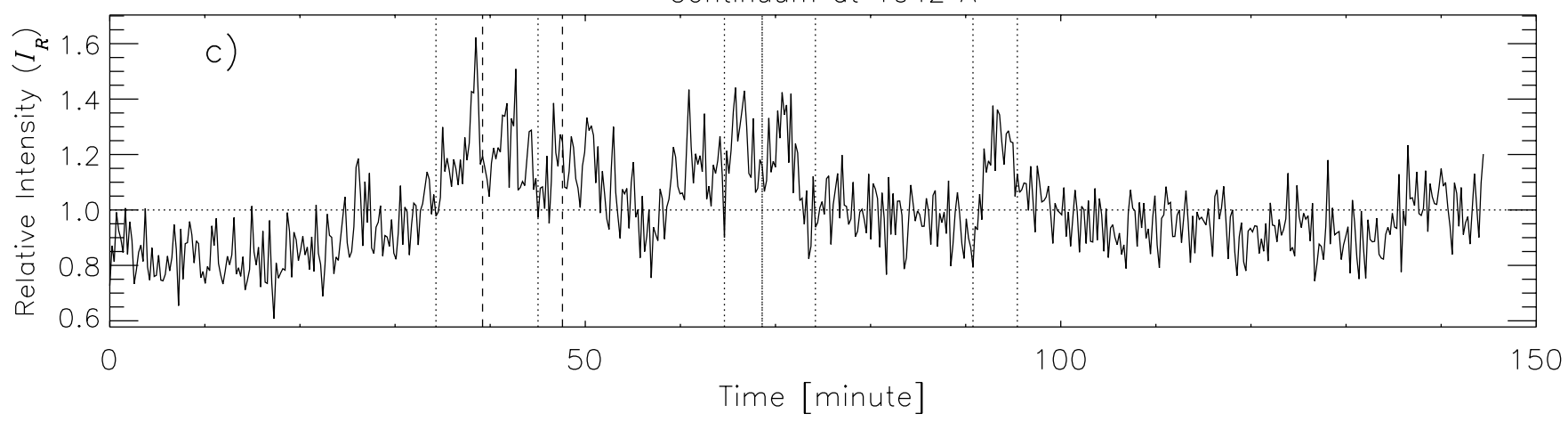

Fig. 3. Sample light curves in $\mathrm{O}_{\text {VI }}$ and $\mathrm{C}_{\text {II }}$ displaying one, respectively four, events classified as blinkers. The continuum light curve is also shown. Light curves are recorded on 16 May 1998. The vertical axes show intensities relative to the averaged background intensity. The horizontal lines signify the following: dotted: the value of the background intensity after iterative removal of the blinker contributions; dashed: the value of the background increased by $1 \sigma$, i.e. by its standard deviation; solid: the threshold intensity used to identify blinkers. The vertical dashed lines in a) and b) represent the start and end times of the blinkers. The areas of the shaded regions are proportional to the energies radiated by the blinkers, and numbers in "white" areas are average enhancements, i.e. ratios between the radiated and "background" energies (see text).

Figures $3 \mathrm{a}$ and $3 \mathrm{~b}$ show light curves of a pixel which we use to describe the four steps of our method of identifying blinkers. Step 1: for each pixel we first determine the time steps at which the intensity is above a given threshold that is chosen to be some factor times the time-averaged intensity of that pixel (solid horizontal lines in Figs. 3a and 3b). For the CDS data analysed by Brković et al. (2001) this threshold was different for each spectral line. In this study the threshold was kept the same for both $\mathrm{C}_{\mathrm{II}}$ and $\mathrm{O}_{\mathrm{VI}}$ reflecting the fact that enhancements in the two lines were of the similar strength. Step 2: starting from the local maximum lying above the threshold we follow the intensity both backward and forward in time, until local minima are found which lie below the time-averaged intensity of the pixel in question (termed the background intensity; $\left.I_{\text {back }}\right)$. These points are designated as the preliminary start and end times of a brightening event. Step 3: if one or more brightness events are identified in a particular pixel a new background intensity is calculated by averaging the intensities of only those time steps not harbouring such events. Steps 1-3 were then repeated with the new (lower) background intensity until no new event is identified. This was the case after less than five iterations for almost all spatial pixels in our data sets. As an illustration in Fig. 3 a threshold of 1.8 leads to the identification of one blinker in $\mathrm{O}$ vi and four blinkers in $\mathrm{C}$ II. Figure 3c shows the corresponding continuum light curve. From now on all intensities are given relative to this final background intensity and are designated as $I_{R}$ in Fig. 3. Finally, in Step 4 we determine the standard deviation $(\sigma)$ of the intensity at the time-steps not harbouring brightness events (i.e. of the background intensity) and we repeat Step 2, but this time looking for local minima below the final background intensity plus the $1 \sigma$ standard deviation above this value (horizontal dashed lines in Fig. 3). This further step was introduced since in a number of cases brightness events with extremely long lifetimes were 
found, which only had a single relatively short-lived peak lying above the threshold, however. Since in most such cases the intensity just fails to drop below the background relatively close to the peak we decided that these long lifetimes are often an artifact of noise or intrinsic, weak background variability, and introduced Step 4.

The contours in the middle and right image in Fig. 2 outline all the points which harbour blinkers during the three hours of observations. The images show that blinkers occur in the network and the inter-network. Since some brightness events started before the beginning or ended after the end of our observations, as in the example in Figs. $3 a$ and $3 b$, their starting and ending times cannot be determined and consequently they are not retained for further analysis (such examples are also present in the bright network near 20", 40" and 80" in Fig. 2).

Please note that the blinkers often show multiple peaks. This is a general feature and not special to our data set. This has already been shown by Brković et al. (2001), who pointed out the existence of simple and complex blinkers without going into deeper analysis. Both SUMER (before and after the spatial resolution reduction) and CDS show the presence of multiple peaks. We are presently analysing the same data sets including information on velocities and noticed that there are several "classes" of blinkers, with and without multiple peaks, amongst others. With that analysis we want to investigate how these different "classes" of blinkers are related to line widths and shifts.

The parameter of interest is the average blinker enhancement, $\bar{E}$, defined as the ratio of the radiated and "background" energies during a blinker event. The radiated energies are shaded regions in Figs. 3a and 3b, while the background energies are "white" regions bounded by vertical dashed lines, the background intensity and the light curve. We use the term "background" only to point out that it relates to the part of the light curve below the background intensity. The blinker enhancement $\bar{E}$ is defined as the excess emission during a blinker lasting from time $t_{0}$ to $t_{1}$ compared to the averaged intensity $<J>$ during the whole time series, i.e.

$\bar{E}=\frac{\int_{t_{0}}^{t_{1}}(J-<J>) \mathrm{d} t}{<J>\left(t_{1}-t_{0}\right)}$.

Using the relative intensities $I_{\mathrm{R}}=J /<J>$, i.e.

$<I>=1$, for the time series we have

$\bar{E}=\frac{\sum_{i=1}^{N}\left[\left(I_{\mathrm{R}}\right)_{i}-1\right]}{N}$,

where the $\left(I_{\mathrm{R}}\right)_{i}$ are the intensities of the line emission of the $i$ th spectral frame and the blinker lasted for $N$ time steps, i.e. was observed for $N$ exposures. Defining the excess intensity as $\tilde{I}_{i}=\left(I_{\mathrm{R}}\right)_{i}-<I>=\left(I_{\mathrm{R}}\right)_{i}-1$, Eq. (2) reads

$\bar{E}=\frac{\sum_{i=1}^{N} \tilde{I}_{i}}{N}$

The average blinker enhancements for detected blinkers in Figs. 3a and $3 \mathrm{~b}$ are given in "white" regions of the same figure.

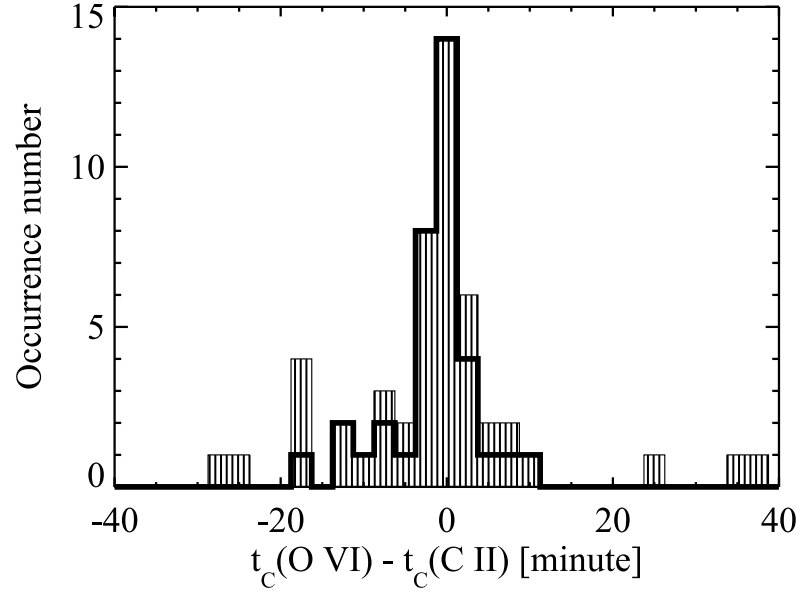

Fig. 4. Differences between "temporal centres" of $\mathrm{O}_{\text {VI }}$ and $\mathrm{C}_{\text {II }}$ blinkers. The thin solid histogram represents time lags of both nearestneighbour blinkers and simultaneous blinkers. The latter are shown as a thick solid histogram.

\section{1. "Simultaneous" blinkers}

We defined "simultaneous" blinkers as those cases in which blinkers observed in two lines in the same spatial location overlap in time by more than $50 \%$ of their individual lifetimes. In the examples in Fig. 3 overlaps amount to $65 \%$ for the $\mathrm{O}$ vi blinker 1 and $53 \%$ for the $\mathrm{C}_{\text {II }}$ blinker 2. Another way to check "simultaneousness" of blinkers in the two lines is to define the temporal centre, $t_{\mathrm{C}}$, for each of these events, i.e. the first moment of the light curve,

$t_{\mathrm{C}}=\frac{\sum_{i=1}^{N}\left(\tilde{I}_{i} t_{i}\right)}{\sum_{i=1}^{N} \tilde{I}_{i}}$.

The difference between $t_{\mathrm{C}}(\mathrm{O} \mathrm{VI})$ and $t_{\mathrm{C}}(\mathrm{C}$ II $)$ can be suitable for testing the time separation of simultaneous blinkers. These differences are plotted in Fig. 4 as a histogram (thick solid). The histogram is centred around zero, with $75 \%$ of the time lags in the range $(-5,5) \mathrm{min}$. The large differences are due to extreme cases when one blinker has a much longer lifetime than the corresponding one in the other line. In such cases the lifetime of the shorter living blinker is completely inside the longer lasting blinker.

We extended the comparison also to differences between nearest-neighbour blinkers, where nearest means in time. In this case we searched for time lags between blinkers which are closest in time when simultaneous blinkers are excluded. Blinkers 1 and 3 in Figs. 3a and 3b form one such example. Note that some blinkers can be accounted as simultaneous and as nearest neighbour. Time lags of solely nearest neighbours are overplotted in Fig. 4 as excesses above the thick solid histogram. The majority, $86 \%$, of these excesses has a time lag above five minutes. If we combine both, simultaneous and nearest-neighbour results, and exclude differences larger than $40 \mathrm{~min}, 90 \%$ of the time lags are below $20 \mathrm{~min}$; of these $80 \%$ belong to simultaneous blinkers. We find that these results support our definition of simultaneous blinkers and establish the spatial and temporal co-existence of blinkers in the lower (C II) and upper ( $\mathrm{O}$ vI) transition region. 


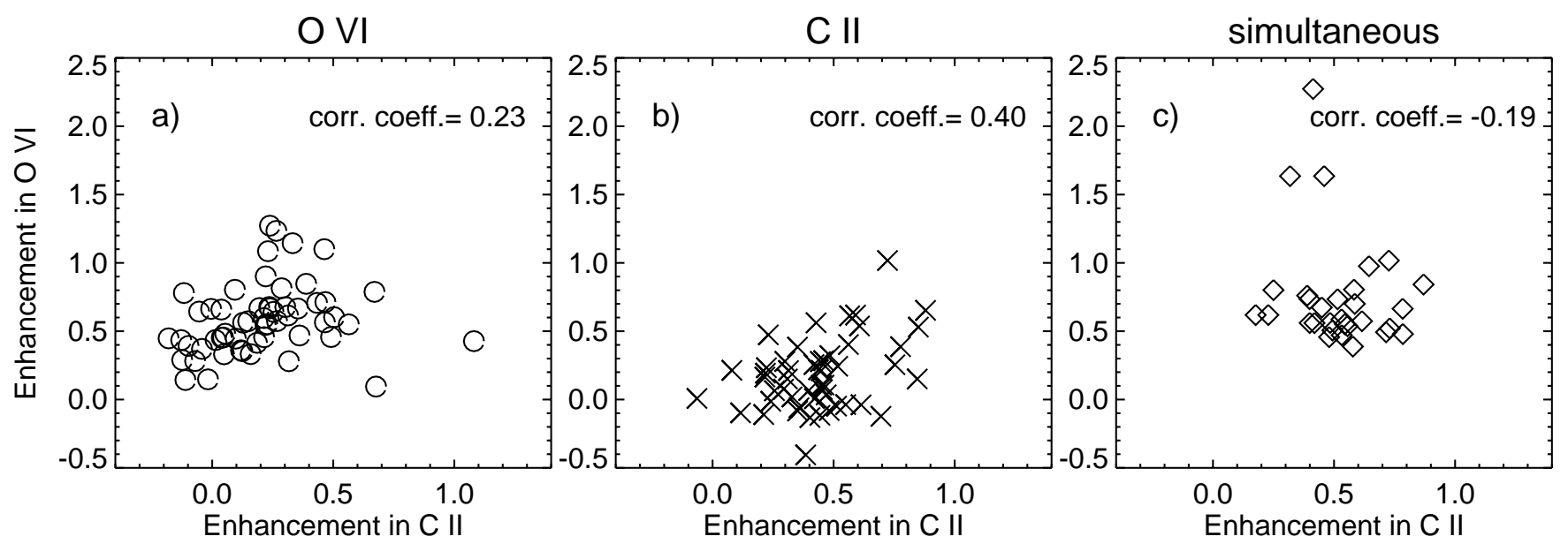

Fig. 5. $\mathrm{C}_{\text {II }}$ and $\mathrm{O}_{\text {VI }}$ enhancements. Circles, crosses and diamonds stand for blinkers originally detected in $\mathrm{O}_{\text {VI }}(\circ), \mathrm{C}_{\text {II }}(\times)$ and simultaneous blinkers $(\diamond)$, respectively. Correlation coefficients of enhancements between two lines are given in the plots.

\section{Analysis of enhancements}

The evidence for blinkers in the lower transition region was shown by Tarbell et al. $(2000)^{2}$, but in our opinion the authors did not point to this fact explicitly enough. In their Fig. 7 enhancements in $\mathrm{C}_{\mathrm{II}}$ are as high as a factor of five, which is comparable to enhancements detected in the $\mathrm{O} v$ and $\mathrm{O} v \mathrm{vi}$ transition region lines. Brković et al. (2001) and Bewsher et al. (2002) reported on brightenings in the $\mathrm{He}_{\mathrm{I}} 584.3 \AA$ line, but not as strong as those found in $\mathrm{OV}$. $\mathrm{He}_{\mathrm{I}}$ is optically thick and influenced by coronal radiation, and as such is not the best representative of chromospheric conditions. The $\mathrm{C}_{\mathrm{II}}$ line reflects well conditions in the lower transition region and, as shown in Fig. 3b, clearly reveals the existence of blinkers. Moreover, from the figure we notice that in some cases during a blinker in the lower transition region there is no significant brightening in the upper transition region while a good signature of the event is present in the low chromosphere.

\section{1. $C$ II and $O$ vı blinkers}

For the blinker detection we applied a threshold of 1.8 and in three data sets we detected $100 \mathrm{O}$ VI and $92 \mathrm{C}_{\text {II }}$ blinkers. The choice of threshold was constrained with the signal-to-noise ratio and the total number of blinkers. Lower thresholds give more blinkers leading to a larger statistical sample, but also to a larger uncertainty in the determination of start and end times. On the other hand, higher thresholds give fewer blinkers. In both cases the choice of the threshold does not significantly influence the final results. When a blinker was found in one line we inspected the behaviour of the other line and the continuum in the same space-time coordinate (location, start and end times). This means that for a blinker detected in $\mathrm{O}_{\mathrm{VI}}$ its average enhancement was determined, as well as enhancements

2 JOP 72 data (a part of our data set) are also analysed by Tarbell et al. (2000). They consider $\mathrm{C}_{\text {II }} 1037 \AA$ as the chromospheric line, even though it is formed above $2 \times 10^{4} \mathrm{~K}$ (Sect. 2) and therefore is from the lower transition region. in $\mathrm{C}_{\mathrm{II}}$ and the continuum for the same space-time coordinate. A similar procedure was applied for blinkers detected in $\mathrm{C}_{\text {II. }}$.

Figure 5 shows $\mathrm{O}_{\text {VI }}$ and $\mathrm{C}_{\mathrm{II}}$ enhancements. Circles (o, Fig. 5a) designate blinkers originally detected in $\mathrm{O}$ VI with the corresponding enhancement in $\mathrm{C}_{\text {II }}$, crosses $(\times$, Fig. 5 b) blinkers originating in $\mathrm{C}_{\mathrm{II}}$ with the corresponding enhancement in $\mathrm{O}$ VI and diamonds $(\diamond$, Fig. 5c) simultaneous blinkers. Note, that simultaneous blinkers are not plotted in Figs. 5a and 5b. Positive (negative) values mean that the blinker light curve is mainly above (below) the average level during a blinker.

In general, the average enhancements in the two lines have similar values, although those of $\mathrm{O}$ VI are somewhat higher. We used the same $x$ - and $y$-ranges for all three plots of Fig. 5 to get a better impression of the distribution of enhancements. In all three plots we recognize the prevalence of positive values (for simultaneous blinkers all values are positive, of course). Correlation coefficients (given on the plots) of enhancements between the two lines are small and absolute values are always less than 0.4. It should be noted that there are cases when a blinker occurs in one line, while the other line shows a decrease in intensity! We determined percentages of negative enhancements in $\mathrm{C}_{\mathrm{II}}$ for blinkers detected in $\mathrm{O}_{\mathrm{VI}}$ and vice versa. These amount to $20 \%$ (26\%) for $\mathrm{C}_{\text {II }}(\mathrm{O}$ vI) enhancements in Figs. 5a and $5 \mathrm{~b}$, respectively. As yet it is unclear whether this effect is of physical importance or just due to chance.

The distributions of the enhancements in Fig. 5 show no trend, i.e. the data points form a kind of cloud. This almost random distribution is also reflected through low correlation coefficients. Application of higher thresholds of 1.9 and 2.0 gives even lower correlation coefficients of 0.02 and -0.19 , respectively for the plot in Fig. 5a, while in other plots the correlation does not change significantly. We notice that enhancements in $\mathrm{O}_{\mathrm{VI}}$ are generally larger than those in $\mathrm{C}_{\mathrm{II}}$, with some exceptions (as in Fig. 3). The highest correlation between enhancements is found for blinkers originating in $\mathrm{C}_{\text {II }}$ (Fig. 5b). A visual inspection of the plot confirms a modest, but not significant, trend in the distribution of data points. Out of 192 blinkers 72 (36 in each line) are simultaneous blinkers (as defined in Sect. 3.1), corresponding to about 38\%. This result points to 

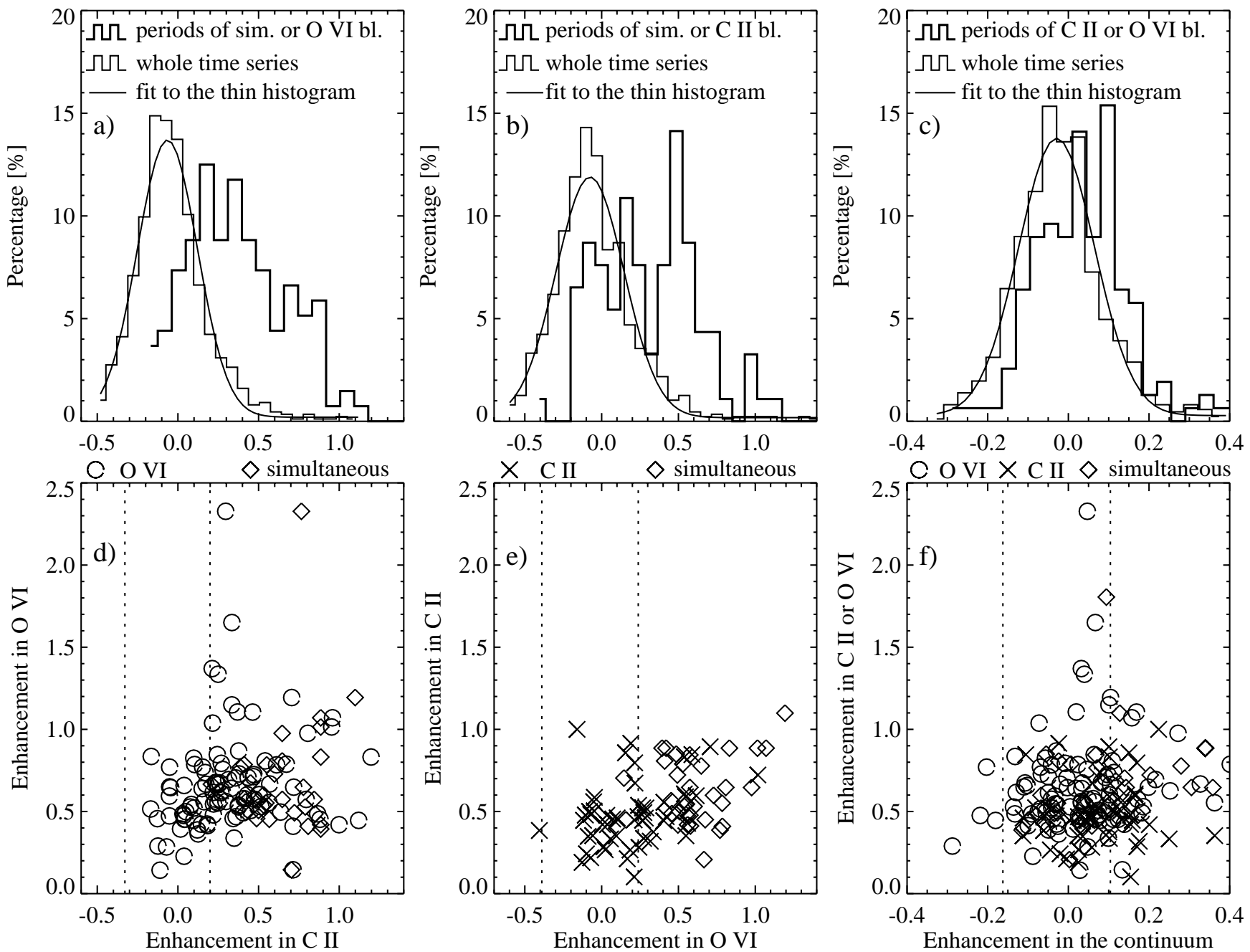

Fig. 6. Enhancements in $\mathrm{C}_{\mathrm{II}}$ and $\mathrm{O}_{\mathrm{VI}}$ and the continuum. Thin histograms show random enhancements over ten minutes intervals. Fits to the thin histograms are represented with Gaussian distributions. Thick solid histograms show enhancements during blinkers in the two lines. The vertical dotted lines represent $1 \sigma$ levels. In the lower panels the symbols are the same as in Fig. 5.

some closer connection between the lower and upper transition region during a blinker event.

To further investigate the relationship between $\mathrm{C}_{\mathrm{II}}$ and $\mathrm{O}$ vi blinkers we determined the average enhancements in the two lines. For this we cut time-series of the two lines into ten minutes intervals, i.e. the average transition region blinker lifetime. We did so for all three data sets and for each of these intervals we evaluated the average enhancement. Enhancements are shown in Figs. 6a and 6b as thin histograms. These provide a sort of "blind test", i.e. the distribution of enhancements when almost noblinker is present. Histograms are approximated by Gaussian distributions plotted as thin solid lines, and the distributions are parametrized with half linewidths, i.e. $\sigma_{1 / \mathrm{e}}$, of 0.23 and 0.26 for $\mathrm{C}_{\text {II }}$ and $\mathrm{O}$ VI, respectively and the mean, $v$, of approximately zero for both lines. This shows that when considering the full time series the enhancements (fluctuations) have a more or less Gaussian distribution, as expected.

Next we restricted this analysis to those time intervals where blinkers are detected. This is shown in Figs. 6d and 6e which are joint plots of Figs. 5a and 5c, respectively
Figs. 5b and 5c. Vertical dotted lines represent $1 \sigma$ levels. Blinkers that show enhancements $|\bar{E}| \geq 1 \sigma$ are considered as reliable. The plots of enhancements change substantially when restricted to blinkers, and instead of near-Gaussian distributions, the enhancements (intensity fluctuations) in the two lines during blinkers are strongly asymmetric (Figs. 6a and 6b, thick solid histograms). On average the fluctuations during $\mathrm{O}$ vi, respectively $\mathrm{C}_{\mathrm{II}}$, blinkers are $42 \%$ enhancement in $\mathrm{C}$ II, respectively $45 \%$ enhancement in $\mathrm{O}$ vi. Deviations of the fluctuations during the blinkers compared to the full time series are significant and clearly evident. The impression is stronger than what one would estimate from Fig. 5 and the correlation coefficients. These results show a connection between blinkers visible in the lower and upper transition region.

\subsection{Relation to the continuum}

Since we did not detect "intrinsic" continuum blinkers, we discuss the response of the continuum to the $\mathrm{O}_{\mathrm{VI}}$ and $\mathrm{C}_{\mathrm{II}}$ blinkers in the same manner as in the case of the two lines (Sect. 4.1). We first wanted to get an idea about average enhancements 
in the continuum in general. Average enhancements of ten minutes intervals are shown in Fig. $6 \mathrm{c}$ as a thin solid histogram which is again of Gaussian shape. The parameters of the Gaussian are: $\sigma_{1 / \mathrm{e}}=0.12, v \approx 0$. As for $\mathrm{C}_{\text {II }}$ and $\mathrm{O}_{\mathrm{VI}}$ this shows that when considering the full time series the continuum enhancements (fluctuations) have a more or less Gaussian distribution.

The distribution of continuum enhancements during blinkers either in $\mathrm{C}_{\mathrm{II}}$ or in $\mathrm{O}$ VI is asymmetric and non-Gaussian (Fig. 6c). This is similar to $\mathrm{C}_{\mathrm{II}}$ and $\mathrm{OVVI}_{\mathrm{V}}$ enhancements (Fig. 6a and b), but less pronounced. The average continuum fluctuations during blinkers show an enhancement of about 0.07. Compared to the width of the enhancement distribution of $\sigma_{1 / \mathrm{e}}=0.12$, this seems not to be significant. However, to get an error estimate of the mean enhancement one has to consider the standard deviation of the mean, $\sigma_{v}=$ $\sigma / \sqrt{N}$, (Bevington \& Robertson 1992), which is only 0.02 . This accounts also for the number of data points (i.e. blinkers) in the statistical analysis. Thus the average enhancement of the continuum during blinkers detected in $\mathrm{C}_{\mathrm{II}}$ and $\mathrm{O}_{\mathrm{VI}}$ is $7 \pm 2 \%$. This shows that there is a signature of blinkers visible in the low chromosphere, even though the signature is only weak.

Like Figs. 6d and 6e, Fig. 6f shows continuum enhancements during $\mathrm{C}_{\mathrm{II}}$ and $\mathrm{O}$ VI blinkers. Distributions of reliable data points, four below and 49 above the $1 \sigma(=0.12)$ level (vertical dotted lines), show no trend. The majority (93\%) of the points in this reliable statistical sample have positive values. We find this result important and expect a modest relationship between the continuum and lower and upper transition region during strong brightenings taking place in $\mathrm{C}_{\text {II }}$ or $\mathrm{O}$ VI. This is supported by Fig. 3 (at time $\approx 40 \mathrm{~min}$ ) where it is clear that the low chromosphere "reacts" during blinkers. The problem, however, remains to understand the deeper meaning of this connection.

\subsection{Duration}

Figure 7 shows histograms of blinker durations detected in the $\mathrm{C}_{\text {II }}$ and $\mathrm{O}_{\text {VI lines. The plots reveal that the } \mathrm{C}_{\text {II }} \text { durations }}$ are much shorter than the $\mathrm{O}$ vi durations. The average durations amount to 10.0 and $6.7 \mathrm{~min}$ for $\mathrm{O}$ VI, respectively $\mathrm{C}_{\mathrm{II}}$ blinkers. The result for $\mathrm{O}$ vi blinkers is comparable to the previously determined average duration of about $15 \mathrm{~min}$. Almost one third of $\mathrm{C}$ II blinkers have lifetimes below three minutes making the median $\mathrm{C}_{\text {II }}$ duration four minutes. In $\approx 20 \%$ of cases two or three blinkers lasting less than three minutes take place one after the other, which could be due to the detection scheme. The rest of the shortest-lived blinkers, as well as the longer-lived ones, were well determined. To preserve the objectivity of the study we did not correct the $\mathrm{C}_{\text {II }}$ duration plot. The effect of the correction would be a shift of the data points to longer durations and a slight increase of the average value.

\section{Conclusion and summary}

In our attempt to better understand the part of the solar atmosphere extending from the low chromosphere to the upper transition region we analysed simultaneous time series spectra

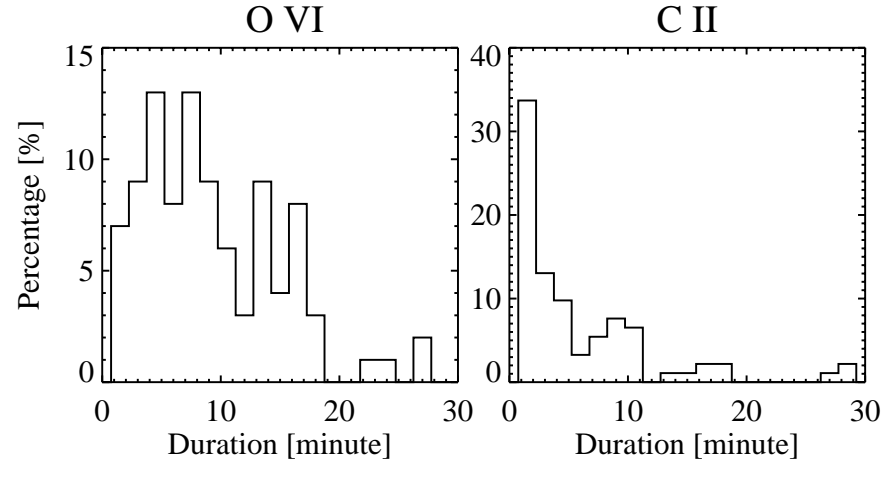

Fig. 7. Durations of $\mathrm{O}_{\text {VI }}$ and $\mathrm{C}_{\text {II }}$ blinkers.

of $\mathrm{C}_{\text {II, }} \mathrm{O}$ VI and the $\mathrm{C}_{\text {I }}$ continuum at $1042 \AA$. We established the existence of blinkers in the lower transition region ( $\mathrm{C}$ II) with enhancements similar to those of upper transition region ( $\mathrm{O} v \mathrm{I})$ blinkers. This shows that blinkers exist on a temperature scale extending over one order of magnitude. Blinkers detected in the lower and upper transition region quite often take place almost simultaneously, which may be a good indication for a connection between these two layers of the solar atmosphere during the blinkers.

Considering the behaviour of the low chromosphere during a blinker event using the $\mathrm{C}_{\mathrm{I}}$ continuum (Sect. 4.2), the situation is less clear than when comparing the lower and upper transition regions. The detected signature in this part of the solar atmosphere during blinkers is weak, but still noticeable. Visual inspection of light curves shows that in some cases the low chromosphere closely "follows" in time the lower and upper transition region (Fig. 3). Based on the statistics of the enhancements in the $\mathrm{C}_{\mathrm{I}}$ continuum we can also conclude that there is a trend for the chromospheric emission to be enhanced (stronger) during a blinker (Sect. 4.2; Fig. 6). This result would partially support the work of Priest et al. (2002) who proposed blinker mechanisms connecting the chromosphere, which is expected to react to the compression of granules, and transition region during a blinker event. Although we can not distinguish between different blinkers mechanisms proposed by Priest et al. (2002), we can establish that blinkers are not exclusively transition region, and eventually coronal, phenomena, but also have traces in the chromosphere. The reason why we do not always see a signficant signature of the blinker in the chromosphere might simply be due to the filling factor. A funnel-like structure which "harbours" a blinker event is significantly (spatially) less present in the low parts of the atmosphere than it is in the upper layers in the transition region.

Consideration of blinker durations showed that $\mathrm{O}$ vi blinkers have an average lifetime of $10.0 \mathrm{~min}$, which is similar to the previously established average lifetime of $\mathrm{O} v$ blinkers of $15 \mathrm{~min}$. $\mathrm{C}_{\mathrm{II}}$ blinkers live on average $6.7 \mathrm{~min}$.

The main result of this paper is that for the first time we could establish a connection of transition region transients, namely blinkers, and the low chromosphere. Further studies will have to show what the physical processes behind this connection are. 
Acknowledgements. We are grateful to the SUMER team and its open data policy. The SUMER project is financially supported by DLR, CNES, NASA and the ESA PRODEX programme (Swiss contribution). SOHO is a mission of international cooperation between ESA and NASA. We would like to thank Reiner Hammer for fruitful discussions and Jo Bruls for discussions on the origin of chromospheric lines and continuua. The work of $\mathrm{AB}$ was supported by the Deutsche Forschungsgemeinschaft grant No. PE 782, which is gratefully acknowledged.

\section{References}

Arnaud, M., \& Rothenflug, R. 1985, A\&AS, 60, 425

Bevington, P. R., \& Robinson, D. K. 1992, Data Reduction and Error Analysis for the Physical Sciences (New-York: McGraw-Hill) Bewsher, D., Parnell, C. E., \& Harrison, R. A. 2002, Sol. Phys., 206, 21

Brković, A., Solanki, S. K., \& Rüedi, I. 2001, A\&A, 373, 1056

Brković, A., Solanki, S. K., \& Rüedi, I. 2002, A\&A, 385, 257

Brueckner, G. E., \& Bartoe, J.-D. F. 1983, ApJ, 272, 329

Chae, J., Wang, H., Goode, P. R., et al. 2000, ApJ, 528, L119

Charbonneau, P. 1995, ApJS, 101, 309

Dere, K. P., Bartoe, J.-D. F., \& Brueckner, G. E. 1984, ApJ, 281, 870
Fontenla, J. M., Avrett, E. H., \& Loeser, R. 1993, ApJ, 406, 319

Handy, B. N., Acton, L. W., Kankelborg, C. C., et al. 1999, Sol. Phys., 187,229

Harrison, R. A., Sawyer, E. C., Carter, M. K., et al. 1995, Sol. Phys., 162,233

Harrison, R. A. 1997, Sol. Phys., 175, 467

Harrison, R. A., Lang, J., Brooks, D. H., \& Innes, D. E. 1999, A\&A, 351,1115

Innes, D. E., Inhester, B., Axford, W. I., \& Wilhelm, K. 1997, Nature, 386,811

Innes, D. E. 2001, A\&A, 378, 1067

Madjarska, M. S., \& Doyle, J. G. 2002, A\&A, 382, 319

McIntosh, S. W., Diver, D. A., Ireland, J., et al. 1998, A\&AS, 132, 145

Parnell, C. E., Bewsher, D., \& Harrison, R. A. 2002, Sol. Phys., 206, 249

Priest, E. R., Hood, A. W., \& Bewsher, D. 2002, Sol. Phys., 205, 249

Scherrer, P. H., Bogart, R. S., Bush, R. I., et al. 1995, Sol. Phys., 162, 129

Tarbell, T. D., Ryutova, M., \& Shine, R. 2000, Sol. Phys., 193, 195

Teriaca, L., Madjarska, M. S., \& Doyle, J. G. 2002, A\&A, 392, 309

Vernazza, J. E., Avrett, E. H., \& Loeser, R. 1981, ApJS, 45, 635

Wilhelm, K., Curdt, W., Marsch, E., et al. 1995, Sol. Phys., 162, 189

Winebarger, A. R., Updike, A. C., \& Reeves, K. K. 2002, ApJ, 570, L105 